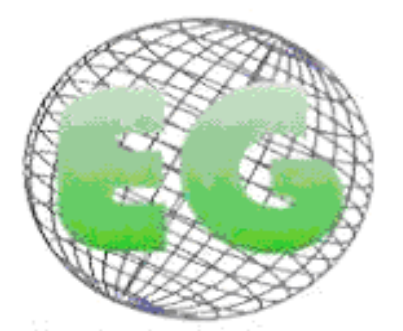

ISSN 1695-6141

$\mathbf{N}^{\circ} 25$
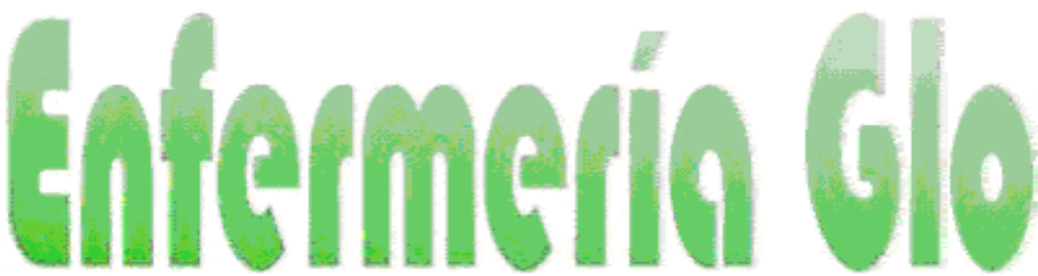

Revista electrónica trimestral de Enfermería

www.um.es/egloball

Enero 2012

\title{
CLINICA
}

\section{Vínculos de los Clientes oncológicos y familiares: una dimensión para ser observada}

Bonds of oncologic clients and relatives: a dimension to be known

\section{*Vestena Zillmer, JG., " ${ }^{* *}$ Schwartz, E., ${ }^{* * * B}$ Burille, A., * "Linck, C de L., **Lange, C., ${ }^{* * * *}$ Eslabão, $\mathrm{A}$.}

*Mestre em Enfermagem. Integrante do Núcleo de Condições Crônicas e suas Interfaces (NUCCRIN).

**Enfermeira Doutora docente da Faculdade de Enfermagem e Obstetrícia da UFPel, Coordenadora da pesquisa; Integrante do Núcleo de Condições Crônicas e suas Interfaces (NUCCRIN). ***Acadêmica de Enfermagem do $8^{\circ}$ semestre Faculdade de Enfermagem e Obstetrícia da UFPel - bolsista do Núcleo de Condições Crônicas e suas Interfaces (NUCCRIN). ****Acadêmica do $3^{\circ}$ semestre da Faculdade de Enfermagem e Obstetrícia da UFPel. Integrante do Núcleo de Condições Crônicas e suas Interfaces (NUCCRIN). Pelotas. Brasil.

(Pesquisa financiada pela Fundação de Amparo à Pesquisa do Estado do Rio Grande do Sul (FAPERGS), sob o n 05/2279.2 PROADE 3, e coordenada pela Prof. Dra. Eda Schwartz, líder do Núcleo de Condições Crônicas e suas Interfaces (NUCCRIN) da Faculdade de Enfermagem e Obstetrícia da Universidade Federal de Pelotas (UFPel). Foi desenvolvida no período de março de 2006 a julho de 2008.$)$

\section{Palabras clave: enfermería; oncología; radioterapia; enlaces. \\ Palavras-chave: enfermagem; oncologia; radioterapia; vínculos \\ Keywords: nursing; oncology; radiotherapy; bonds}

\section{RESUMEN}

Introducción: El perfil de la población brasileña ha ido cambiando con el aumento de enfermedades crónicas. Entre ellos, está el cáncer que ocupa la segunda causa de muerte por enfermedad en Brasil, siendo considerado un grave problema de salud pública.

Objetivos: Conocer a los bonos que los clientes y sus familias establecen en la vivencia con el cáncer. 
Metodología: La investigación cualitativa, exploratoria y descriptiva. Dieciocho clientes y dos familiares participaron en el estudio. Fue usada la entrevista semi-estructurada, con la redacción de genograma y ecomapa.

Resultados: En el discurso de los sujetos, surgieron dos grupos temáticos: el fortalecimiento de los vínculos y lazos rotos por la enfermedad y tratamiento.

Conclusión: Los resultados ayudaron a reflexionar sobre el cuidado de enfermería en el tratamiento con radioterapia del cáncer y, en colaboración con los posibles cambios en la práctica de la enfermera en oncología cerca de estos clientes.

\section{RESUMO}

Objetivos: Conhecer os vínculos que os clientes oncológicos e seus familiares estabelecem ao vivenciar o câncer.

Metodologia: Pesquisa qualitativa, exploratória e descritiva. Participaram dezoito clientes em tratamento radioterápico e dois familiares. Utilizou-se a entrevista semi-estruturada, com elaboração de genograma e ecomapa.

Resultados: No discurso dos sujeitos, emergiram dois núcleos temáticos: vínculos fortalecidos e vínculos rompidos pela doença e tratamento.

Conclusões: Os resultados contribuíram para refletir sobre cuidado de enfermagem no âmbito do câncer e do tratamento radioterápico, colaborando com possíveis mudanças na prática de cuidar da enfermeira em oncologia junto a esses clientes.

\section{ABSTRACT}

Aims: To ascertain the bonds that the oncologic clients and their relatives establish when living with cancer.

Methodology: a qualitative, exploratory and descriptive research. Eighteen clients in radiotherapy treatment and two relatives participated in the research. The semi-structured interview was used, with the elaboration of genogram and ecomap.

Results: In the speech of the subjects, two thematic nuclei emerged: strengthened bonds and bonds broken by the disease and treatment.

Conclusions: The results contributed to considerations on nursing care inthe radiothreapytreatment of cancer, and in collaboration with possible changes in the nurse in care oncology close to these clients.

\section{INTRODUCCIÓN}

A lo largo de los años, el perfil de la población brasileña, con el aumento de las condiciones crónicas, viene modificándose y presentando un serio desafío para el sistema de salud. Entre ellos está el cáncer, que ocupa la segunda causa de muerte por enfermedad en Brasil, siendo considerado un grave problema de salud pública ${ }^{\left({ }_{1}\right)}$. Esta es una de las enfermedades más temidas por las personas, y mucho más que una afección biológica, se caracteriza como un conjunto de sentimientos ambivalentes cargados de significados.

En ese sentido, el cáncer es considerado una enfermedad simbólica, en la medida en que trae consigo significaciones diversas tales como: desorden, castigo, siendo relacionado con la muerte ${ }^{(2,3)}$. Al tener un diagnóstico de cáncer, enfermedad que evoca sufrimiento y preocupaciones, ocurre una desestructuración no sólo del paciente sino también de su 
familia que es sorprendida por un momento de estrés, el cual conlleva cambios en la dinámica familiar ${ }^{(4)}$.

De ese modo, el equipo de salud precisa conocer los vínculos que se establecen en respuesta de la enfermedad y su tratamiento y, de esta manera, tener condiciones de evaluar, planear e intervenir, para cuidar de la persona portadora de cáncer y de su unidad familiar. Con esto, proporcionará cuidados que mejoren su calidad de vida y promuevan el fortalecimiento de las relaciones y potencialidades de la familia.

Las interrelaciones que la familia establece con sus diversos ambientes, microsistema, mesosistema, exosistema e macrosistema, son fundamentales para que ocurra el desenvolvimiento humano ${ }^{(5,6)}$. Es a partir de la red de relaciones que los vínculos con vecinos, comunidad, trabajo, escuela y Servicio de Salud, podrán, o no, potencializar el proceso de desenvolvimiento de la persona con cáncer y su familia en la realización y búsqueda de prácticas de cuidados.

El vínculo se constituye en una importante estrategia para adhesión y calidad de cuidados prestados al cliente y familia. Se entiende por vínculo de apoyo, aquella persona que puede participar de dos o más sistemas, formando un engranaje, lo que facilita el proceso de resolución de crisis desarrolladas generadas por la familia ${ }^{(56)}$. A partir de lo expuesto, este estudio tiene como objetivo conocer los vínculos que los clientes oncológicos y sus familiares establecen al vivir el cáncer y el tratamiento radioterapéutico.

\section{METODOLOGÍA}

Se trata de un estudio descriptivo, con enfoque cualitativo y con un abordaje ecológico, realizado con pacientes portadores de cáncer y sus familiares, atendidos en el Ambulatorio de Radioterapia de una Universidad Pública de la región sur de Brasil, que es referencia en tratamiento contra el cáncer. Participaron de la investigación dieciocho pacientes que estaban en tratamiento radioterapéutico y dos familiares. La colecta de los datos ocurrió en el periodo de Marzo del 2006 a Diciembre del 2008, en la sala de espera del referido servicio. Como instrumentos de recolecta de datos, fueron utilizados la entrevista semiestructurada o genograma y el ecomapa.

El genograma es un árbol familiar, representando la estructura familiar interna y el ecomapa es la representación visual de las relaciones entre los miembros de la familia y los sistemas más amplios. Estos posibilitan la percepción de toda la familia, delineando su naturaleza, las interrelaciones y puntos intermedios, puentes para construir y recursos para ser buscados y movilizados para conflictos ${ }^{(7)}$.

Los datos fueron colectados entre los meses de Marzo de 2006 a Diciembre de 2008, por medio de una entrevista semiestructurada. Las entrevistas fueron grabadas y realizadas en el Ambulatorio de Radioterapia. Los datos de las grabaciones fueron transcritos y agrupados por semejanza ${ }^{(8)}$ formando núcleos temáticos.

El proyecto fue encaminado y aprobado con el número 028/86 por el Comité de Ética en Pesquisa de la Faculdade de Medicina de la Universidade Federal de Pelotas, según los principios y la resolución del Conselho Nacional de Saude $n^{\circ}$ 196/96. Al acordar el participar del estudio, los sujetos de la investigación firmaron el acuerdo de consentimiento libre y claro. Para mantener el secreto y el anonimato, los clientes y familiares fueron nombrados por número arábigo, obedeciendo a la secuencia de las entrevistas, sumada la edad del cliente, teniendo su familiar el mismo número. 


\section{RESULTADO Y DISCUSIÓN}

Participaron de este estudio clientes y familiares que frecuentaban un Ambulatorio de Radioterapia. Las declaraciones de los entrevistados fueron agrupadas en núcleos temáticos, teniendo en cuenta el objetivo de este estudio. De ese modo, emergieron dos núcleos, sumadores de significados, que sirvieron de base para orientar la discusión: Los vínculos fortalecidos y los vínculos rotos por la enfermedad.

\section{Los vínculos fortalecidos}

Algunos de los clientes expresan en sus declaraciones el fortalecimiento de los vínculos, principalmente, en el microsistema familiar, por parte de los hijos, cónyuge y padres. La enfermedad no involucra solamente a la persona enferma si no a todo el grupo familiar y puede servir como unificadora de la familia. La familia, ante la enfermedad, parece extender la mano en dirección al enfermo y se articula para ayudarlo ${ }^{(3)}$.

Los vínculos con la familia se solidificaron constituyendo una fuente esencial de apoyo, para la mayoría de los clientes en tratamiento radioterapéutico. En la mayoría de las veces, todo sistema familiar se moviliza para cuidar del proceso de tratamiento del cáncer. Otro aspecto importante es que, aun estando la familia lejos, esta se hace presente, usando diversas maneras de comunicación para estar más próximo de su familiar. Esto se hizo evidente en otro estudio con mujeres diagnosticadas con cáncer $^{(9)}$. Las declaraciones siguientes expresan la complicidad familiar:

\section{[...] los padres se preocupan ...] llaman y cuando pueden vienen y se quedan conmigo ....] (004, 28 años - cliente)}

]...] mi familia siempre estuvo muy unida ]] hijos, hermanos, todos unidos ]] y ahora cada vez más, porque cada uno quiere dar un apoyo a su manera, entonces, queda más unida todavía (094, 48 años-cliente)

La familia es una importante fuente de apoyo, ya que ella es quien va a convivir con el familiar enfermo, compartiendo sus limitaciones y pérdidas, buscando confortar en las horas difíciles $^{(10)}$. Evidenciamos en este estudio que la familia realiza cuidados relacionados con la higiene, la alimentación, estar presente para ir al médico y al Ambulatorio de Radioterapia, proporcionando seguridad, cuidados y solidaridad en el discurrir del tratamiento.

[...] mis cuidados se multiplicaron con la higiene, limpieza y agotadora para mí, pero yo estoy sintiéndome muy bien porque estoy consiguiendo hacer lo que yo siempre quise, y él está dejándose ayudar, eso es muy importante, porque él nunca quiso [...] (003 65 años-hija);

[...] ellos (familia) me cuidan mucho, mis remedios, mi alimentación [...](002, 74 años-cliente)

En el estudio con familiares de mujeres con cáncer de mama, se identificó que el cáncer tiene la capacidad de provocar alteraciones en la funcionalidad familiar y que la familia, ante las situaciones que precisan vivir, se sirven de los potenciales de cada miembro para alcanzar la estabilidad del contexto familiar ${ }^{(11)}$. El querer estar más cerca de su familiar y dedicarse a él más tiempo es una de las prioridades establecidas de la familia, cuando se depara con una enfermedad como el cáncer. 
Se puede constatar una menor referencia a los vínculos fortalecidos con relación a la red social. Algunos de los clientes relataron que encontraron ánimo y amparo en la religión, en la iglesia y en amigos, como personas con quienes comparten sus anhelos y dudas. En relación a los amigos, percibimos que, en aquellos que son verdaderamente amigos, las relaciones permanecen y se fortalecen con el surgimiento del cáncer, proporcionando ánimo al familiar enfermo y a su familia.

\section{[...] converso bastante con una amiga mía [...] (004, 28 años-cliente)}

[...] tengo dos hijos, conversamos sobre la enfermedad, me dan apoyo, converso con ellos y con mis amigas cuando las encuentro (120-72 años-cliente)

\section{[...] hablo sobre eso (cáncer) con una amiga (002- 74 años-cliente)}

El cuidado prestado por la familia es fortalecido también por la red social, formada por parientes, amigos y vecinos ${ }^{(12,13)}$. Compartir angustias, dudas y posibilidades con personas próximas, hace que familiares y clientes se sientan acogidos y aliviados. Tener con quien contar en una hora de dificultades trae un sentimiento de seguridad, pues saben que tienen un soporte y pueden recurrir a él, constituyéndose en un vínculo de apoyo. Resaltamos que la familia, que posee una red social, afronta mejor las adversidades impuestas por la enfermedad y el tratamiento.

[...] me gusta frecuentar mi religión[...] con la esperanza grande, si Dios quiere[...] (003,65 años - cliente);

La convivencia con sentimientos de desesperanza lleva muchas veces a la reflexión sobre la actual situación y las posibilidades de futuras ${ }^{(14)}$. En este contexto, la espiritualidad ocupa un espacio importante en la vida de las personas y es co-participante en el lidiar con la enfermedad. Creer en algo superior vuelve el tratamiento menos penoso y renueva las fuerzas de quien está en una lucha diaria por sobrevivir.

La fe es muchas veces vista como un "medicamento" muy poderoso para los clientes con cáncer, pues estos mantienen la esperanza en busca de un significado y es de confianza en un ser superior. Esto genera fortaleza y serenidad para aceptar la enfermedad ${ }^{(15)}$. Por eso, se puede decir que la espiritualidad, la fe, es una herramienta fundamental para luchar contra la enfermedad y tener una mejor calidad de vida, tanto para el cliente oncológico como para la familia.

\section{Los vínculos rotos por la enfermedad}

Cuando el cliente portador de cáncer y su familia no poseen una red de apoyo, con la llegada de la enfermedad las dificultades se suman a las diarias ${ }^{(16)}$. Una enfermedad como el cáncer puede interferir y fragilizar los vínculos anteriormente establecidos. Fue observado, en este estudio, que con la enfermedad surgieron fragilidades sociales, tales como: de trabajo, tiempo libre y de amistades.

[...] dejar la casa, marido e hijo para venir y hacer el tratamiento [...] salgo el domingo y vuelvo el sábado (004,28 años-cliente)

[...] antes yo y mi familia teníamos muchos amigos [...] y ahora ellos ya no lo son [...] (005,38 años-cliente); 
El tratamiento no siempre está disponible en la localidad en la que vive la persona acometida, como en este caso, en el que la cliente necesita trasladarse de una región a otra. Además de esto, hay dificultades financieras, de locomoción y de dejar hijos, marido y la rutina doméstica en busca de una supervivencia mayor.

La enfermedad grave, por sí misma, genera una serie de sentimientos y situaciones que pueden desestabilizar una familia, intensificándose con la separación de sus miembros. Permanecer lejos de la familia en un momento tan difícil genera sufrimiento y ansiedad para el cliente, y la familia se siente impotente, por no poder acompañar su familiar o por no poder estar a su lado en ese momento.

[...] el trabajo es lo que más mudó [...] y nosotros viajábamos, pero ahora no lo hacemos más. (006, 66 años-cliente)

Cuando ocurre un cambio en la familia después de la interferencia de la enfermedad, hay una alteración para una nueva posición de equilibrio ${ }^{(7)}$, como la convivencia con los amigos, vecinos, fiestas y tiempo libre. Puntualizamos que, muchas veces, ocurre la ruptura de las comodidades cotidianas y se buscan nuevas formas de adaptación. Así, la familia busca encontrar la mejor manera de equilibrarse en una situación de desequilibrio ${ }^{(3)}$.

Algunos de los clientes y familiares expresaron que no pueden trabajar más, realizar actividades de tiempo libre y pasan a aislarse de la convivencia social. Las declaraciones muestran cómo la enfermedad interfirió en la vida de los clientes portadores de cáncer y su familia. Muchos vínculos fueron rotos, por imposición de la enfermedad y por falta de esclarecimientos respecto del diagnóstico. Esto puede ser debido a la fragilidad de una red social de apoyo.

[...] paré de ir al Centro de Tradición Gaucha, trabajo de cestería y de cachepó de periódicos, por causa de eso (cáncer) [...] (007, 76 años-cliente)

Perdí el vínculo con las personas de la vecindad [...] algunos quedaron distantes después de que quedaron sabiendo que era cáncer, creo que ellos tienen miedo de contraerla [...] dejé de trabajar, de ir a fiestas (004, 28 años-cliente)

El rechazo social está íntimamente ligado al medio, los estigmas y los preconceptos ${ }^{(17)}$. La enfermedad crónica provoca, por momentos, relaciones de aislamiento que llevan al cliente muchas veces a la soledad y a la depresión, ocasionando rupturas de relaciones duraderas y significativas para este ${ }^{18}$. Un ejemplo es la imagen corporal que induce a las personas a quedarse con miedo, principalmente de enfermedades infectocontagiosas, lo que en este caso no corresponde a la realidad, haciendo con que el cliente quede cada vez más excluido de la sociedad.

\section{hija)}

[...] Ahora el no va más a fiestas, no va al fútbol [...] antes él iba (003,65 años-

El cáncer todavía es una enfermedad muy estigmatizada y, frecuentemente, asociada a la "terminalidad". Por muchos, es visto como algo que se debe esconder, por lo que estar enfermo puede llevar a la discriminación y al rechazo social, desde el ámbito familiar hasta en el trabajo del individuo, que, además de tener que enfrentar la enfermedad, necesita enfrentar el descrédito social ${ }^{(18)}$. En la sociedad contemporánea, en la cual el individuo es explotado de forma mercantil, la pérdida de capacidad productiva en razón de 
una enfermedad, hace que el desamparo social sea acusado con más intensidad por el paciente ${ }^{(19)}$.

[...] mudó bastante, el negocio del servicio, que ella siempre me ayudaba, hay yo tuve que parar con las cosas para atenderla a ella [...] (006-66 años-esposo)

Enfatizamos que el cáncer es una enfermedad envuelta en muchas mitificaciones y miedos, que no ocasionan apenas consecuencias físicas y psíquicas a su portador. Estos acarrean numerosos cambios en su vida social, llevando, muchas veces, al cliente a quedar aislado, distante de las actividades que antes le daban placer, y hasta abandonar el trabajo. Además de enfrentar el preconcepto de la sociedad, clientes y familiares, muchas veces, tienen que luchar contra sus propios preconceptos y valores que fragilizan las relaciones y vínculos en el proceso de rehabilitación y cuidado.

\section{CONSIDERACIONES FINALES}

El cliente portador de cáncer en tratamiento radioterapéutico rompe las estructuras y las relaciones sociales de lo cotidiano, además abre "nuevas ventanas", como nuevas amistades, readaptaciones familiares. Aunque la enfermedad sea una vivencia individual, los que la comparten mantienen los lazos que son fortalecidos.

De esa manera, una enfermedad grave puede interferir y fragilizar los vínculos anteriormente establecidos, pero también puede intensificar los vínculos ya existentes y traer nuevos vínculos a la familia y al cliente. Sobre este enfoque, el mirar sobre esta población debe de estar anclado en el conocimiento de la dinámica de las relaciones dando a los profesionales de la salud, especialmente los de enfermería, un importante papel que desarrollar.

Por tanto, es fundamental que los profesionales de salud conozcan la naturaleza de los vínculos, la red de apoyo utilizada, fragilizada o rota, siendo también de su responsabilidad emprender fuerzas para ayudar en el establecimiento de estrategias de manejo con el cáncer y el tratamiento, garantizando una mejor calidad de vida para los clientes y sus familias.

\section{REFERÊNCIAS}

1. Brasil Ministério da Saúde. Estimativas 2008: Incidência de Câncer no Brasil. Instituto Nacional de Câncer: 94 p. 2007.

2. Silva LC. Câncer de mama e sofrimento psicológico: aspectos relacionados ao feminino. Psicologia em Estudo 2008 abril-junho; 13 (2):231-237.

3. Bielemann VLM. Uma experiência de adoecer e morrer em família. In: Elsen I, Marcon SS, Silva MRS, organizadoras. O viver em família e a interface com a saúde e a doença. 2.ed. Maringá: Eduem; 2004. p.199-215.

4. Barros DO, Lopes RLM. Mulheres com câncer invasivo do colo uterino: suporte familiar como auxílio. Rev Bras Enferm. 2007 maio-junho; 60(3):295-298.

5. Bronfenbrenner U. A ecologia do desenvolvimento: experimentos naturais e planejados. Porto Alegre: Artes Médicas, 1996.

6. Schwartz E. O viver o adoecer e o cuidar das famílias de uma comunidade rural do extremo Sul do Brasil: uma perspectiva ecológica. 2002. 202 p. Tese (Doutorado em Filosofia da Enfermagem) - Programa de Pós-Graduação em Enfermagem, Universidade Federal de Santa Catarina, Florianópolis. 
7. Wright L., Leahey M. Enfermeiras e famílias: um guia para avaliação e intervenção na família. 4 ed. São Pulo: Roca, 2009.

8. Minayo MCS. O desafio do conhecimento: pesquisa qualitativa em saúde. São Paulo: Hucitec. 2007.

9. Molina MAS; Marcon SS. Mudanças nos relacionamentos com os amigos, cônjuge e família após o diagnóstico de câncer na mulher. Rev. Bras. Enferm. 2006; 54 (1): 514-520. 10. Volpato FS, Santos GRS. Pacientes oncológicos: um olhar sobre as dificuldades vivenciadas pelos familiares cuidadores. Imaginário 2007; 13 (14): 511-544.

11. Biffi RG. A dinâmica familiar de um grupo de 14 mulheres com câncer de mama. [tese]. Ribeirão Preto (SP): Universidade de São Paulo. Escola de Enfermagem; 2003.

12. Budó MLD, Resta DG, Denardin JM, Ressel LB, Borges ZN. Práticas de cuidado em relação à dor - A cultura e as alternativas populares. Esc Anna Nery Rev Enferm. 2008 março; 12(1): 90-96.

13. Gutiérrez MGR, Arthur TC, Fonseca SM, Matheus MCC. O câncer e seu tratamento: impacto na vida dos pacientes. Online Brazilian Journal of Nursing, 2007 6(0)

14. Ferreira NML, Chico E, Hayashi VD. Buscando compreender a experiência do doente com câncer. Rev. Ciênc. Méd. 2005 maio-junho; 14 (3): 239-248.

15. Teixeira JJV, Lefèvre F. Significado da intervenção médica e da fé religiosa para o paciente idoso com câncer. Ciência \& Saúde Coletiva 2008; 13 (4): 1247-1256.

16. Ribeiro NRR. A família enfrentando a doença grave da criança. In: Elsen I, Marcon SM, Silva MRS. O viver em família e sua interface com a saúde e a doença. 2 ed. Maringá: Eduem, 2004. p.183-198.

17. David GLB. A teoria de king: um referencial para o cuidado de famílias convivendo com Aids. In: Elsen I, Marcon SM, Silva MRS. O viver em família e sua interface com a saúde e a doença. 2 ed. Maringá: Eduem, 2004. p.341-343.

18. Linard AG, Silva FAD, Silva RM. Mulheres submetidas a tratamento para câncer de colo uterino - percepção de como enfrentam a realidade. Revista Brasileira de Cancerologia 2002; 48 (4):493-498.

19. Santos PAL. A doença crônica incapacitante e dependente na família. 2003. 20p.

Dissertação (Mestrado em Saúde Pública) Escola Nacional de Saúde Pública, Lisboa.

ISSN 1695-6141

(c) COPYRIGHT Servicio de Publicaciones - Universidad de Murcia 